\title{
Use of Acoustic Energy in the Processing of Molten Aluminium Alloys
}

Hélder Puga $^{1 a}$, Sónia Costa ${ }^{1, b}$, Joaquim Barbosa ${ }^{1, c}$ and Carlos Silva Ribeiro, ${ }^{2, d}$

${ }^{1} \mathrm{CT} 2 \mathrm{M}$ - Centre for Mechanical and Materials Technologies, Universidade do Minho, 4800-058 Guimarães, Portugal

\author{
${ }^{2}$ FEUP, Departamento de Engenharia Metalúrgica e de Materiais, 4200-465 Porto, Portugal \\ apuga@dem.uminho.pt, bsonia.costa@plastifa.pt, ${ }^{\mathrm{c}}$ kim@dem.uminho.pt, ${ }^{\mathrm{d}}$ sribeiro@fe.up.pt
}

Keywords: Processing; Aluminium Alloys; Ultrasonic; Mechanical Properties

\begin{abstract}
During the last years aluminium alloys have been gaining increased acceptance as structural materials in the automotive and aeronautical industries, mainly due to their light weight, good formability and corrosion resistance. However, improvement of mechanical properties is a constant in research activities, either by the development of new alloys or by microstructure manipulation.

This presentation focuses a novel effective dynamic methodology to perform microstructural refinement / modification and degassing of light alloys, namely aluminium alloys, by applying acoustic energy to the melts.

High intensity acoustic energy significantly improves the microstructure, therefore the mechanical properties of those alloys, avoiding the use of traditional chemically based degassing and refining techniques which are less effective and present significant environmental impact. Ultrasonic (US) vibration has proven to be extremely effective in degassing, controlling columnar dendritic structure, reducing the size of equiaxed grains and, under some conditions, producing globular grains and modifying the eutectic silicon cells in Al-Si alloys. The mechanisms of US processing of aluminium melts are discussed and experimental results on this field are presented.
\end{abstract}

\section{Introduction}

Al-Si based casting alloys with small amounts of $\mathrm{Cu}$ and/or $\mathrm{Mg}$ are among the most commonly used foundry alloys for high strength light weight applications. Although the beneficial effect of $\mathrm{Cu}$ and $\mathrm{Mg}$ in mechanical strength, their presence has a detrimental effect in the alloys ductility, since in the presence of $\mathrm{Fe}$ they promote the development of brittle $\mathrm{Mg}$-rich and $\mathrm{Cu}$-rich intermetallics, that are usually difficult to eliminate.

$\mathrm{AlSi} 7 \mathrm{Mg}$ and $\mathrm{AlSi} 9 \mathrm{Cu} 3$ are among the most used $\mathrm{Al}$ alloys for industrial applications. The ascast microstructure of these consist of coarse dendritic $\alpha$-Al solid solution phase and Al-Si eutectic where Si is usually present in the form of brittle large plates with sharp sides and ends that act as stress concentrators decreasing ductility. Intermetallic phases like the eutectics $\mathrm{Al}_{2} \mathrm{Cu}$ and $\mathrm{Mg}_{2} \mathrm{Si}$, "Chinese script" shaped $\mathrm{Al}_{15}(\mathrm{Mn}, \mathrm{Fe})_{3} \mathrm{Si}_{2}$ and long and sharp needles of $\mathrm{Al}_{5} \mathrm{FeSi}$ may also precipitate in the interdendritic or intergranular regions. This microstructure, together with traditional casting defects like porosity and inclusions is strongly detrimental to the alloy tensile and fatigue properties [1-3].Thus, casting defects control and microstructure refinement/modification are imperative to achieve high mechanical performance in Al castings.

Grain refinement is directly related to the number of active nuclei in the melt. The traditional approach to increase nucleation is by adding master alloys like Al-Ti-B, that release large quantities of $\mathrm{TiAl}_{3}$ and $\mathrm{TiB}_{2}$ particles in the melt, which are easilly enveloped by the $\alpha$-Al phase during cooling, promoting heterogeneous nucleation. However, chemical refinement presents several drawbacks, namely inability to avoid dendritic structures and refine intermetallic phases (although promoting a better distribution) and inability to decrease the grain size to values below $200 \mu \mathrm{m}$ [4]. 
Eutectic Si modification is crucial to achieve high mechanical properties, and is considered much more important than grain refinement itself [5]. Al-Sr master alloys and $\mathrm{Na}$ based fluxes are usually used to change Si morphology from acicular to a fine fibrous structure increasing the alloy ductility, but increase the amount of porosities in castings [6] and generate great amount of dross and smoke.

The current major problem associated to Al alloys is the inability to completely eliminate the various production defects and simultaneously obtain a fully ultra refined microstructure to enhance $\mathrm{Al}$ castings performance. Current routes to enhance the mechanical performance of $\mathrm{Al}$ castings are limited to the development of new alloys, namely containing Transition and/or Rare Earth Metals [7,8], new refiners [9] and automatic process control systems [9], which will increase both production and tooling costs. Integrated approaches, combining the principles of traditional casting processes and new melt treatment techniques seem to be interesting and simple but still unexplored routes to enhance the mechanical behaviour of high strength $\mathrm{Al}$ components made of current alloys.

On this work, the potential of acoustic energy to degas AlSi9Cu3 and refine an AlSi7Mg alloy, and their associated mechanisms are presented.

\section{Acoustic Energy in Liquid Metals Processing}

Ultrasonic degassing/refinement/modification is a valuable alternative to replace traditional melt treatment techniques and overtake their associated problems/limitations. When a liquid metal is submitted to high intensity ultrasonic vibrations, the alternating pressure above the cavitation threshold brings about numerous tiny bubbles in the liquid metal, which start growing, pulsing on a continuous expansion/compression regime and finally collapse. During expansion, bubbles absorb energy from the melt, undercooling the liquid at the bubble-liquid interface, resulting in nucleation on the bubble surface. When bubbles collapse acoustic streaming develops in the melt, distributing the nuclei into the surrounding liquid producing a significant number of nuclei in the molten alloy, thus promoting heterogeneous nucleation. If the melt temperature is low enough to avoid dissolution of the embryos, their life time is long enough to promote a high density of nuclei in the melt, thus leading to the development of large number of globular grains with small grain size [10].

During the last years, some researchers, including the authors, developed extensive research on this field, and a US degassing and refinement technique has been developed for Al die-casting. Besides promoting the formation of $\alpha$-Al globular grains with less than $20 \mu \mathrm{m} \mathrm{[11]} \mathrm{and} \mathrm{fully}$ modify the eutectic Si from acicular to globular/fibrous particles less than $5 \mu \mathrm{m}$ long, US processing refines and distributes uniformly the intermetallic phases.

US processing is also an extremely efficient and clean degassing process [12]. When cavitation develops, mass transfer processes intensify, accelerating the diffusion of dissolved hydrogen towards the developed bubbles. As acoustic cavitation progresses with time, adjacent bubbles touch and coalesce, growing to a size sufficient to allow them to rise up through the liquid, against gravity, until reaching the melt surface. Degassing rates can be 4 times higher than traditional gas purging techniques and $98 \%$ of the theoretical alloy density can be achieved after just 1 minute degassing [12], which is virtually impossible with current gas purging techniques.

\section{Experimental Procedures}

Experimental set-ups. The configuration of the ultrasonic set-up depends on the operation to carry on, but there are elements which are always present. Basically, an ultrasonic equipment for treatment of molten alloys consists of an ultrasonic power supply unit, a high power ultrasonic converter, an acoustic waveguide and an acoustic resonator. The waveform generated by the ultrasonic power supply unit is converted in repetitive multi-frequency mechanical vibrations by the ultrasonic converter, which are driven to the acoustic resonator through the waveguide, which also works as a booster. The acoustic load (resonator, liquid, mould, etc) starts producing its own vibration, oscillating in one or more of its natural resonance frequencies.

Traditional ultrasonic applications are based on fixed-frequency, well-tuned ultrasonic sources, where a large number of designs and matching parameters must be respected [10,11]. Extensive 
field tests have demonstrated that in order to achieve high efficiency, the ultrasonic systems must be well tuned to the load [10,13]. Since most ultrasound units work inherently in non-stationary conditions, they have to continuously adapt themselves to the load to maximize the efficiency, which is difficult to achieve with fixed-frequency units. In our case, a novel signal processing technique with the potential to synchronously excite many vibrating modes through the coupled harmonics and sub harmonics in solids and liquid containers to produce high intensity multimode vibrations that are uniform and repeatable, has been used. Such sonic and ultrasonic driving creates uniform and homogenous distribution of cavitation and acoustical activity inside the vibrating system, while avoiding the creation of stationary and standing waves, so that the whole vibrating system is fully agitated.

In Fig. 1, three different set-ups with different purposes are presented. Set-up a) can be used for melt degassing inside the melting crucible. For large volumes of metal, a low frequency mechanical vibrator can be coupled to the radiator through a interface to induce melt stirring and improve degassing [14]. Set-up b) is suitable for grain refinement. The acoustic energy is transmitted to the melt inside the melting crucible for an established period of time at constant temperature, and the metal is then poured into the moulds. Set-up c) is suitable to promote grain refinement and silicon modification. The waveguide is coupled to the mould using suitable clamps and the mould works as a resonator, transmitting the acoustic energy to the alloy during solidification. This set-up has been designed for metallic moulds, and can't be used with sand moulding. A suitable set-up for sand casting has already been developed and successfully evaluated, and results will soon be published.
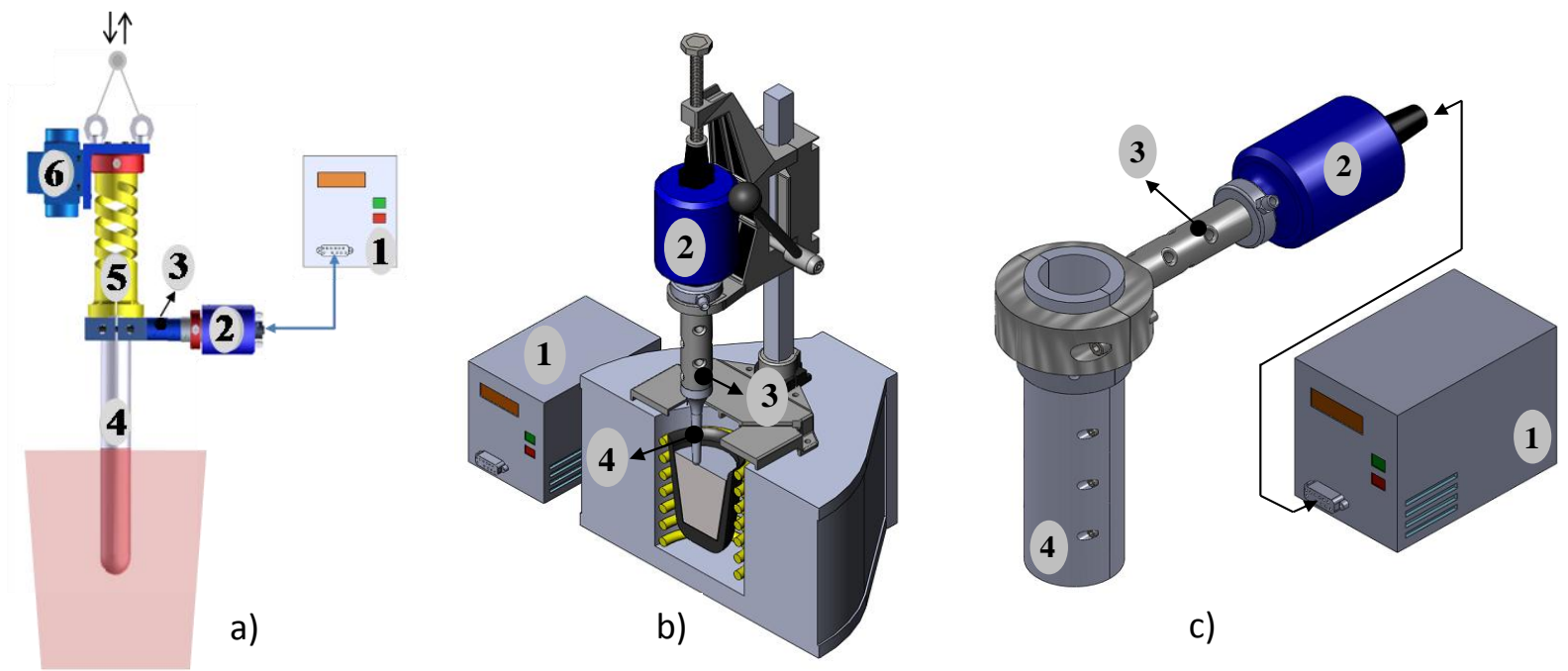

Figure 1 - a) Degassing set-up; b) Refinement set-up; c) Refinement and modification set-up. Main constituents: 1. US power supply unit; 2. US converter; 3. Waveguide; 4. Resonator/Mould; 5. Interface; 6. Mechanical vibrator.

Processing variables. Processing variables in ultrasonic processing of liquid metals are numerous, and difficult to list. On the other hand, their relative importance depends on the operation to carry out, and some of them are crucial for one application, but irrelevant for another. Nevertheless, parameters like ultrasonic frequency and electric energy converted into acoustic energy, geometrical configuration and material of the resonator, waveguide and clamps, relative dimensions of the resonator and volume of liquid to be processed, liquid temperature and processing time are among the most important factors controlling the process efficiency. Furthermore, since the central mechanism of ultrasonic processing of liquid alloys is cavitation, it is easy to understand that those factors that promote the highest cavitation levels will be of importance to the operation. It is known that ultrasound loses its oscillation energy and sound intensity along the path in a melt or in a solid media. If this loss is significant the intensity rapidly falls to the cavitation threshold, and cavitation ceases. This effect, known as sound absorption/attenuation, depends on different parameters, like the melt viscosity, resonator and waveguide temperature, etc, which need to be under control. 


\section{Experimental Results of US Processing of Aluminium Alloys}

Degassing. In Fig.2 the US degassing rates/efficiencies of $\mathrm{AlSi} \mathrm{Cu} 3$ alloys are presented under the form of alloy density variation as a function of processing time, for different US parameters and melt temperatures, and are the results of experiments developed by the authors at the University of Minho with $4 \mathrm{~kg}$ melts. Density evaluation was performed by the Reduced Pressure Test (RPT) and the apparent density measurement method.

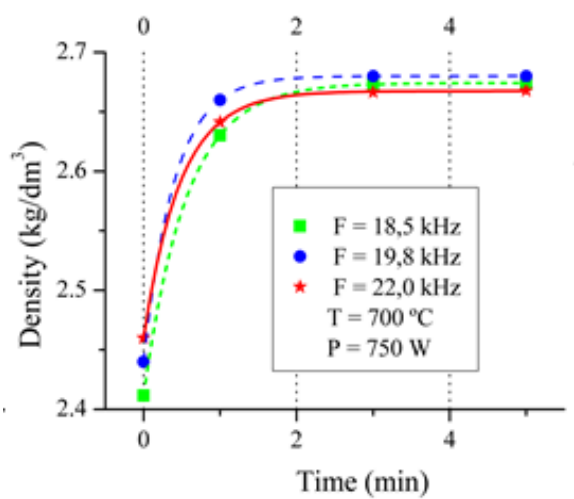

a)

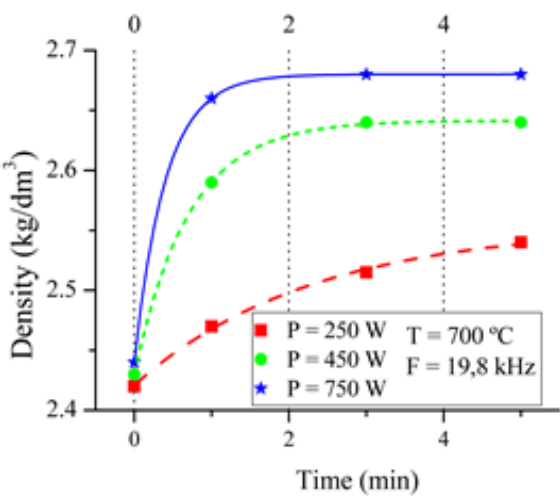

b)

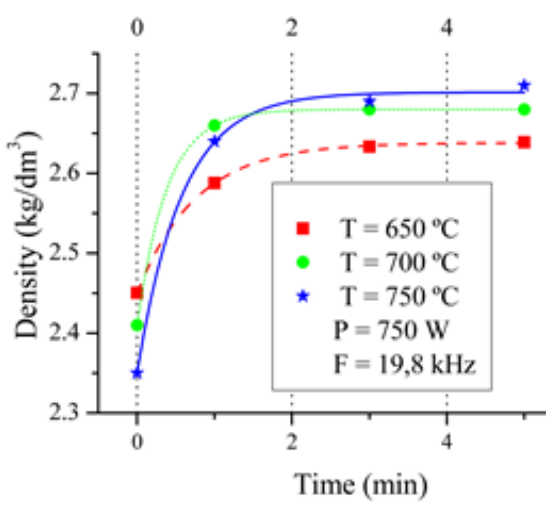

c)

Figure 2 - Density of Alsi9Cu3 alloy as a function of processing time and: a) Melt temperature; b) US frequency; c) Electric power [12]

It is evident from Fig.2a) that US frequency has no apparent influence on degassing efficiency, but the degassing kinetics is time dependent and changes as hydrogen is being removed from the melt. The maximum alloy density (2.68) was obtained after 2 minutes ultrasonic processing (although after 1 minute it was already about $98.5 \%$ of the maximum value), which was a consequence of a high hydrogen removal rate. As US processing continues, density increasing rate slows down meaning that a hydrogen content steady-state plateau is being reached. For longer degassing times the density remains constant, and the difference/balance to the theoretical alloy density is only due to solidification defects. This behaviour is due to the mechanism of hydrogen removal from aluminium melts and the effect of acoustic cavitation on that mechanism. In fact, due to the extremely large number of very small bubbles developed in the molten alloy by cavitation, diffusion of hydrogen towards them is very fast. As a consequence, the number of hydrogen atoms in the molten alloy decreases at the same rate, slowing down the diffusion mechanism of hydrogen, thus degassing and density increasing rates.

However, electric power has a strong influence on the kinetics of hydrogen removal, which was maximum for $750 \mathrm{~W}$, as shown in Fig.2b). The intensity of cavitation increases directly with this parameter [10] and, for the processing conditions used on this work, $750 \mathrm{~W}$ promoted a welldeveloped cavitation regime and the highest density of cavitation bubbles. On these conditions, the partial displacement of drops of liquid in the treated volume develops strong acoustic streams, that improves the coagulation of separate bubbles of hydrogen and its floating to the surface of the pool and, consequently, a raise of the degassing and density increasing rates.

Besides electric power, degassing efficiency also depends on the melt temperature. For temperatures below $750{ }^{\circ} \mathrm{C}$, the degassing rate was found to be sensitive to melt temperature, increasing directly with it (Fig.2c). For melt temperatures of 700 and $750{ }^{\circ} \mathrm{C}$ the hydrogen removal and density increasing rates, as well as the density steady-state plateaus, were quite similar (2.68

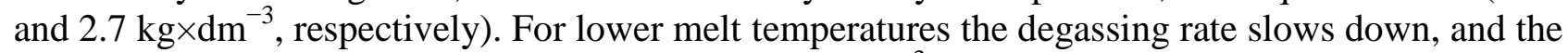

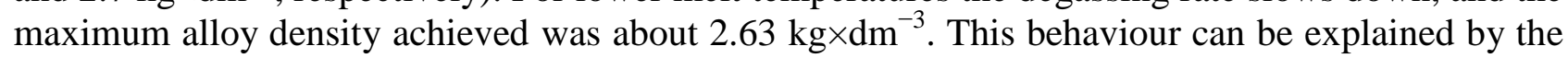
decrease of the melt viscosity with temperature making more difficult the pulsation of cavitation bubbles, their coagulation and floating to the surface. Moreover, the diffusion coefficient of hydrogen in liquid metals decreases as temperatures drops, slowing down the diffusion of hydrogen from the liquid to the cavitation bubbles. 
Microstructure refinement and modification. Figure 3 presents the microstructures of AlSi7Mg alloys ultrasonically processed in different conditions: a) and c) are the resultant microstructures of 1 minute isothermal processing at $630^{\circ} \mathrm{C}$, and pouring into a metallic mould preheated to $250^{\circ} \mathrm{C}$. Cast samples were cylinders with $40 \mathrm{~mm}$ in diameter and $90 \mathrm{~mm}$ long. The US effect on grain refinement and elimination of the traditional dendritic structure is clearly seen. The sample presents mainly homogeneous $\alpha$-Al globular grains with average grain size around $50 \mu \mathrm{m}$ and a few rosette like grains. Although US processing was able to refine the $\alpha$-Al structure, it had no effect on the eutectic $\mathrm{Si}$, which kept the traditional acicular shape of non-modified structures. Isothermal processing at 620 and $640^{\circ} \mathrm{C}$ led to the formation of more heterogeneous structures, with larger $\alpha$-Al grains and higher number of rosettes. This was due to the short survival time of nuclei at $640^{\circ} \mathrm{C}$, and difficulty to develop cavitation at $620^{\circ} \mathrm{C}$ due to the higher melt viscosity. These processing temperatures were selected because they are slightly above the liquidus temperature of the alloy, therefore a localized slight undercooling induced by cavitation could easily promote nucleation.
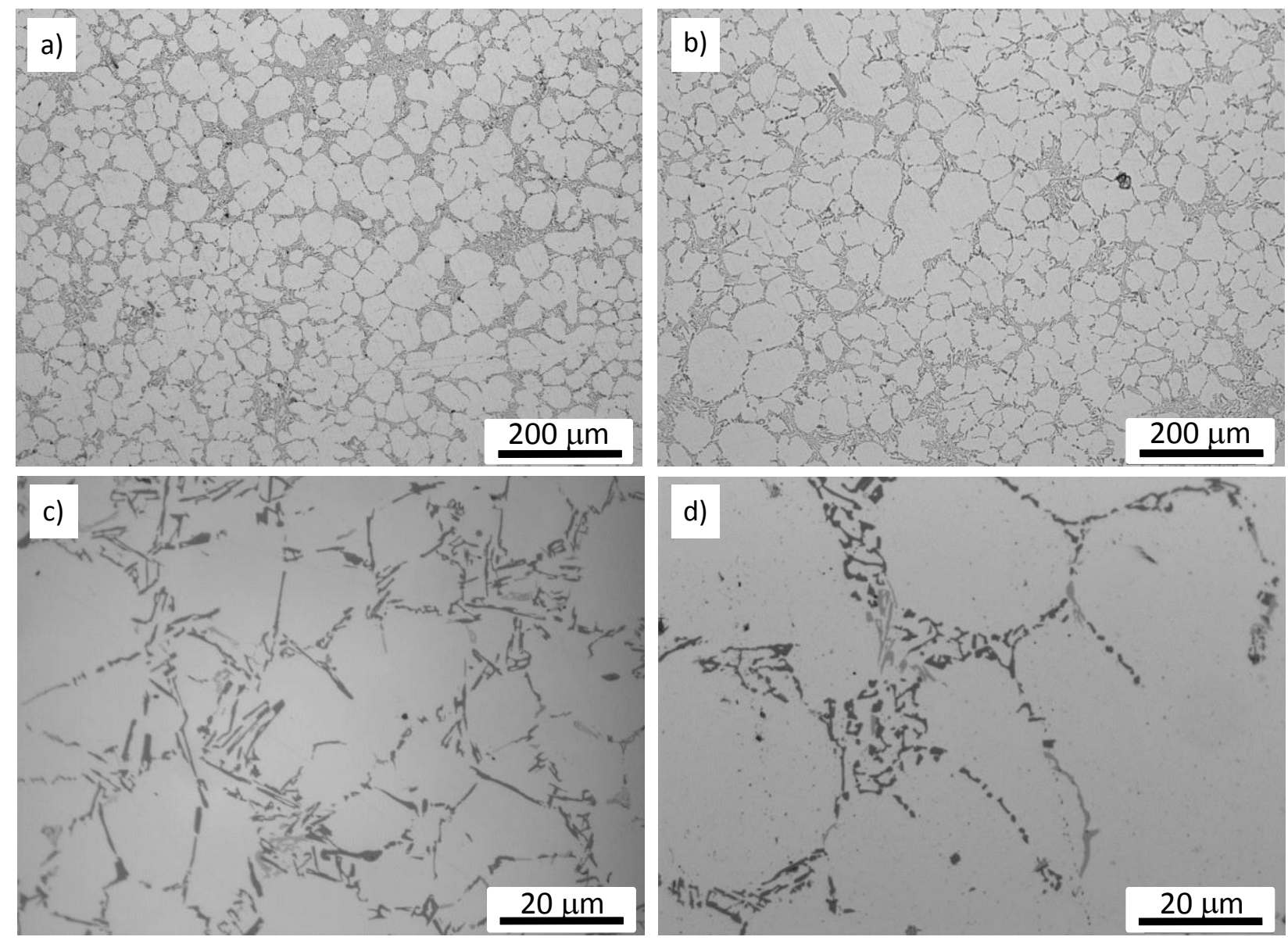

Figure 3 - Microstructure of US processed AlSi7Mg alloy: a) and c) at $630^{\circ} \mathrm{C}$ for 1 minute before pouring; b) and d) during cooling inside a metallic mould, until reaching $585^{\circ} \mathrm{C}$.

Figs.3b) and d) show the microstructure of the same alloy US processed using Set-up c). The alloy was poured at $700^{\circ} \mathrm{C}$ in the same mould pre-heated to $250^{\circ} \mathrm{C}$ and cooled under the influence of US vibration until reaching $585^{\circ} \mathrm{C}$, which is $10^{\circ} \mathrm{C}$ above solidus temperature. Grain refinement is evident, however a more heterogeneous globular structure with different grain sizes coexisting with some rosettes was obtained. In this case, due the high cooling rate of the melt, the number of nuclei developed by cavitation was smaller, therefore grain refinement was not so efficient. Nevertheless, a clear full Si modification occurred, resulting in small particles with average area less than $10 \mu \mathrm{m}^{2}$. Although the mechanism of US Si modification is not fully understood yet, some authors attribute it to acoustically induced convection and pressure variations in the liquid pools, which affect 
nucleation by altering the constitutional supercooling at the front of the growing eutectic grains [15].

US processing also has a strong effect on the Fe containing intermetallic phases in AlSi9Cu3 and AlSi7Mg alloys, namely the "Chinese script" shaped $\mathrm{Al}_{15}(\mathrm{Mn}, \mathrm{Fe})_{3} \mathrm{Si}_{2}$ and the long and sharp needles of $\mathrm{Al}_{5} \mathrm{FeSi}$. Recent experiments developed by the authors [16] showed that US processing changes their morphology into short and thin particles with different shapes, uniformly distributed throughout the matrix.

\section{Conclusions}

The effect of US processing of Al alloys is being extensively studied and results obtained so far show that:

- US treatment promotes degassing and alloy densities close to $98.5 \%$ of the respective theoretical densities are achieved in less than 2 minutes;

- Ultrasonic treatment is an efficient physical technique to control the morphology and size of primary $\mathrm{Al}$ grains, refines and distributes intermetallic phases and promotes eutectic $\mathrm{Si}$ modification; $\alpha$-Al grain sizes less than $50 \mu \mathrm{m}$ and eutectic Si particles with area less than $10 \mu \mathrm{m}^{2}$ can be obtained;

- US processing is not only temperature and time dependent, as well as its efficiency is also determined by the electric power and configuration of the US set-up.

\section{References}

[1] M. Merlin et al., Impact behaviour of A356 alloy for low-pressure die casting automotive wheels. J. Mat. Proc. Tech. 209 (2009) 1060-1073.

[2] K.G. Basavakumar et al., Influence of grain refinement and modification on microstructure and mechanical properties of Al-7Si and Al-7Si-2.5Cu cast alloys. Mat. Char. 59 (2008) 283-289.

[3] Q.G. Wang et al., Fatigue behaviour of A356-T6 aluminium cast alloys. Part I. Effect of casting defects. J. Lig. Met. 1 (2001) 73-84.

[4] G.K. Sigworth, Grain Refinement of Aluminium Casting Alloys AFS Trans. 67 (2) (2007) 1-12

[5] J.A. García-Hinojosa et al., Structure and properties of Al-7Si-Ni and Al-7Si-Cu cast alloys non-modified and modified with Sr. J. Mat. Proc. Tech. 143-144 (2003) 306-310.

[6] S.D. Mcdonald et al., Modification-related porosity formation in hypoeutectic aluminiumsilicon alloys. Met. Mat. Trans. B 35B (2004) 1097-1106.

[7] K.E. Knipling et al., Precipitation evolution in Al-Zr and Al-Zr-Ti alloys during aging at 450$600{ }^{\circ} \mathrm{C}$. Acta Mat. 56 (2008) 1182-119.

[8] M.S. Kaisera et al., Effect of scandium on the microstructure and ageing behaviour of cast Al6Mg alloy. Mat. Char. 59 (2008) 1661-1666.

[9] D. Apelian. Aluminium cast alloys: Enabling tools for improved performance. NADCA, Wheeling, Illinois, 2009.

[10]G.I. Eskin, Ultrasonic treatment of light alloy melts, 1st edition. Gordon and Breach Science Publishers, Amsterdam, 1998.

[11]S. Zang et al., High-energy ultrasonic field effects on the microstructure and mechanical behaviours of A356 alloy. J. All. Comp. 470 (2009) 168-172.

[12]H. Puga et al., The influence of processing parameters on the ultrasonic degassing of molten AlSi9Cu3 aluminium alloy. Mat. Letters 63 (2009) 806-808.

[13]M. Prokic, European Patent Application EP 1238715A1, 2001.

[14] H. Puga et al., The combined effect of melt stirring and ultrasonic agitation on the degassing efficiency of AlSi9Cu3 alloy. Mat. Letters 63 (2009) 2089-2092.

[15]X. Jian et al., Refinement of eutectic silicon phase of aluminium A356 alloy using highintensity vibration. Scr. Mat. 54 (2006) 893-896.

[16]H. Puga et al., Influence of Ultrasonic Melt Treatment on Microstructure and Mechanical Properties of AlSi9Cu3 alloy. J. Mat. Proc. Tech. (2011). Submitted. 